\title{
Annual lung function changes in young patients with chronic lung disease
}

\author{
P.J.F.M. Merkus, H.A.W.M. Tiddens, J.C. de Jongste
}

\begin{abstract}
Annual lung function changes in young patients with chronic lung disease. P.J.F.M. Merkus, H.A.W.M. Tiddens, J.C. de Jongste. C) ERS Journals Ltd 2002.

ABSTRACT: Reference equations for ventilatory function that use different statistical models may introduce artifacts that affect the estimated change of lung function during growth in young subjects. The effect of differently modelled reference equations on the estimated annual change of forced expiratory volume in one second (FEV1) and forced vital capacity (FVC) in young patients with chronic lung disease was assessed.

Four frequently used reference equations were used to describe the longitudinal changes of FEV1 and FVC in 52 patients ( 23 females) with cystic fibrosis (CF) during a mean follow-up of 3.9 yrs.

Choice of reference equations directly affected value and, most importantly, estimated annual change of FVC and FEV1. Mean \pm SD annual change of FEV1 varied from $2.2 \pm 6.2$ to $-2.2 \pm 3.6 \%$ of predicted. For two reference equations the estimated individual changes of FEV1 and FVC in CF were positively correlated with mean individual age. This probably reflects underestimation of deteriorating lung function.

Variability of annual change was independent of age only when reference equations that were designed to accurately predict lung function during the pubertal growth spurt were used. These findings have implications for patient care and clinical research.
\end{abstract} Eur Respir J 2002; 19: 886-891.
Dept of Paediatrics, Subdivision of Respiratory Medicine, Erasmus Medical Centre Rotterdam, Children's Hospital, Rotterdam, The Netherlands.

Correspondence: P.J.F.M. Merkus, Sophia Children's Hospital, Erasmus Medical Centre Rotterdam, PO Box 2060, 3000 CB Rotterdam, the Netherlands.

Fax: 31104636772

E-mail: merkus@alkg.azr.nl

Keywords: Adolescents, chronic lung disease, cystic fibrosis, follow-up, growth spurt, ventilatory function reference equations

Received: June 222001

Accepted after revision November 5 2001
Medium- or long-term follow-up of ventilatory function in children and adolescents requires a correction for lung growth. This correction is not only crucial for monitoring patients with chronic lung disease and to evaluate individual therapeutic interventions, but is also important in longitudinal clinical trials where indices such as forced expiratory volume in one second (FEV1) and forced vital capacity (FVC) are often primary end-points. Correction for lung growth is usually achieved by expressing lung function parameters as a percentage of predicted ( $\%$ pred), although predictions derived from cross-sectional studies may not accurately reflect lung growth within an individual $[1,2]$.

In most pulmonary function reference equations for growing children, standing height is used as a proxy for lung growth. Power functions, exponential functions, and linear functions are used to describe the relationship between standing height and pulmonary function indices [3]. Though explained variance using these equations is high, it has been demonstrated that during the pubertal growth spurt these equations do not describe the development of pulmonary function accurately [4-6]. Because of the phase shift in somatic growth and lung growth, ventilatory function appears to lag behind during the peak of somatic (leg) growth. This can result in overestimation of ventilatory function before and after the growth spurt, and in underestimation of ventilatory function during the growth spurt when expressed as \% pred. Therefore, including age as an additional independent variable in the reference equations would theoretically improve the validity of the prediction, and such reference equations have been developed based on large numbers of children and adolescents from a general population $[4,6]$. However, the implications of this issue for patient care, or for long-term clinical trials in teenagers with chronic lung disease have not been evaluated extensively. In a recent study [7] it was demonstrated that the rate of decline of FEV 1 in cystic fibrosis (CF) patients varied according to each one out of four American reference equations. FEV1 may be the most robust test of airway function, but if correction for lung growth is unreliable, this test is of limited value when studied longitudinally. Accurate correction of ventilatory function for growth is especially important in children and adolescents with chronic lung disease. In this study, the effect of various reference equations [4, 6, 8, 9], that used different models, and lung-function data of European and American children and adolescents, on the estimated long-term change of FEV1 and FVC in Dutch children and adolescents with $\mathrm{CF}$ was assessed. Some of these equations have been used previously in important clinical trials for patients with asthma [10] and in patients with CF $[11,12]$. The hypothesis was that the choice of reference equations would have a considerable effect on the estimated change of FEV1 
and FVC in children and adolescents with chronic lung disease even though their growth spurt is usually delayed and reduced [13, 14].

\section{Materials and methods}

\section{Data set}

The data set consisted of measurements of FEV1 and FVC derived from maximal expiratory flow/ volume curves obtained from all children and adolescents with $\mathrm{CF}$ who attended the outpatient clinic for respiratory medicine between 1994-1999. These measurements were carried out during annual elective measurements, when children were clinically stable. Procedures were carried out using a Lilly-type heated pneumotachograph (Jaeger, Würzburg, Germany) according to international guidelines [15]. Patients were included when at least three measurements were available with $1-\mathrm{yr}$ intervals between measurements. Mean individual age was based on these time points.

\section{Reference equations}

The study focused on FEV1 and FVC, expressed as $\%$ pred. Four types of reference equations were studied. 1) A power function based on height only described by ZAPLETAL et al. [9], expressed as \% pred Z. This was developed using data from 173 subjects aged 6-18 yrs. 2) Linear equations by KNUDSON et al. [8] describing a linear relationship between lung function and height until the age of $12 \mathrm{yrs}$, and a linear relationship with age and height for the range of 12-17 yrs of age, expressed as \% pred K. These were based on a study of 407 healthy subjects aged 6-25 yrs. 3) A more complex power model including age-height interaction by QUANJER et al. [4], expressed as $\%$ pred $\mathrm{Q}$. This was developed using six datasets consisting of ventilatory function data from a total of 5,861 subjects in the age range of 6-21 yrs. 4) A set of age-specific power equations that were based on height only by WANG et al. [6], expressed as \% pred W. These were based on a study in 11,630 healthy subjects aged 6-18 yrs.

The latter two reference equations $[4,6]$ were especially designed to correct, as much as possible, for the effects of the pubertal growth spurt on ventilatory function in adolescents.

\section{Data analyses}

FEV1 and FVC expressed as \% pred Z, \% pred Q, $\%$ pred $\mathrm{K}$ and $\%$ pred $\mathrm{W}$ were compared within individuals using paired t-tests. Linear regression against age was used to calculate annual individual change of FEV1 and FVC (expressed as \% pred). No correction for slope variance or number of measurements was applied, as the focus of the study was not to determine the exact slope of lung function over time, but to assess the within-subject differences depending on the four equations used. Individual slopes were compared using paired t-tests. The age dependency of $\%$ pred $\mathrm{Z}, \%$ pred $\mathrm{Q}, \%$ pred $\mathrm{K}$, and $\%$ pred $\mathrm{W}$ was studied using correlation coefficients of individual slopes against mean individual ages using linear regression.

\section{Results}

In 52 patients (23 females) three or more consecutive annual measurements were obtained. The age ranges for males and females at the start and end of the study were 5.8-16.4 and 8.8-19.5 yrs, respectively. Anthropometric and lung-function parameters at the start and end of the analyses are shown in table 1. FVC values at the start and at the end of the study were similar for $\%$ pred $\mathrm{Z}$ and $\%$ pred $\mathrm{K}$, while mean $\%$ pred $\mathrm{Q}$ was lower $(\mathrm{p}<0.001)$ and $\%$ pred $\mathrm{W}$ was higher than the other values $(\mathrm{p}<0.001)$. At the start and at the end of the study FEV1 was highest when expressed as \% pred $\mathrm{K}$, lower when expressed as \% pred $\mathrm{Z}$ or $\%$ pred $\mathrm{W}$, and lowest when expressed as $\%$ pred Q $(\mathrm{p}<0.001)$.

The mean annual change of FVC and FEV1 varied according to the reference equation used (table 2). When analysed for those children presumably at the start of, or in the pubertal growth spurt (subgroup

Table 1. - Anthropometric and lung function data for 52 cystic fibrosis patients at the start and end of the study

\begin{tabular}{lcc}
\hline & Start & End \\
\hline Height cm & $140.1 \pm 12.6$ & $157.4 \pm 13.5$ \\
Age yrs & $10.3 \pm 2.2$ & $13.8 \pm 2.5$ \\
Weight kg & $32.9 \pm 8.3$ & $45.9 \pm 11.7$ \\
FVC \% pred Z & $96.3 \pm 16.2$ & $94.2 \pm 14.7$ \\
FVC \% pred K & $96.0 \pm 16.1$ & $93.9 \pm 14.4$ \\
FVC \% pred Q & $93.6 \pm 14.3$ & $90.2 \pm 13.6$ \\
FVC \% pred W & $98.5 \pm 15.1$ & $96.5 \pm 13.9$ \\
FEV1 \% pred Z & $89.6 \pm 20.2$ & $83.2 \pm 16.8$ \\
FEV1 \% pred K & $94.2 \pm 19.5$ & $104.1 \pm 21.2$ \\
FEV1 \% pred Q & $84.8 \pm 18.1$ & $77.7 \pm 15.7$ \\
FEV1 \% pred W & $88.7 \pm 18.9$ & $81.6 \pm 16.6$ \\
\hline
\end{tabular}

Data are presented as mean \pm SD. FVC: forced vital capacity; FEV1: forced expiratory volume in one second; Z: using reference equation described in ZAPLETAL et al. [9]; K: using reference equation described in KNUDSON et al. [8]; Q: using reference equation described in QUANJER et al. [4]; W: using reference equation described in WANG et al. [6].

Table 2. - Annual change of forced expiratory volume in one second (FEV 1 ) and forced vital capacity (FVC) in 52 cystic fibrosis patients

\begin{tabular}{lrc}
\hline & \multicolumn{1}{c}{ FEV1 } & FVC \\
\hline$\%$ pred Z & $-2.0 \pm 3.8$ & $-0.8 \pm 3.1$ \\
$\%$ pred K & $2.2 \pm 6.2$ & $-0.8 \pm 3.2$ \\
$\%$ pred Q & $-2.1 \pm 3.4$ & $-1.0 \pm 2.8$ \\
$\%$ pred W & $-2.2 \pm 3.6$ & $-0.7 \pm 3.1$ \\
\hline
\end{tabular}

Data are presented as mean \pm SD unless otherwise stated. Z: using reference equation described in ZAPLETAL et al. [9]; $\mathrm{K}$ : using reference equation described in KNUDSON et al. [8]; Q: using reference equation described in QUANJER et al. [4]; $\mathrm{W}$ : using reference equation described in WANG et al. [6]. 
with age $>11$ yrs, $n=35)$, these differences became more pronounced (table 3 ). Mean annual change of FEV1 was positive, especially in the subgroup aged $>11$ yrs, when expressed as $\%$ pred $\mathrm{K}$. There was a curvilinear relationship between mean age and annual change of $\%$ pred $\mathrm{K}$, with peak values around 12 yrs (fig. 1). On average, annual change of FEV1\% pred K increased with mean age (table 4). The differences between FEV1 \% pred $\mathrm{K}$ and FEV1 \% pred Q were highly variable and had their maximal value $\sim 12$ yrs (fig. 2).

Mean annual changes of FEV1 expressed as \% pred $\mathrm{Z}, \%$ pred $\mathrm{Q}$ and $\%$ pred $\mathrm{W}$ were negative and of similar magnitude, but there was a significant agedependency of these slopes for $\%$ pred $\mathrm{Z}$ but not for $\%$ pred $\mathrm{Q}$ or $\%$ pred W (table 4). Hence, the difference between FEV1\% pred Q and FEV1\% pred $\mathrm{Z}$ varied with age (fig. 2). Annual changes of $\%$ pred $Q$ and $\%$ pred $\mathrm{W}$ did not differ significantly, and no age dependency could be demonstrated for either values of FEV1 and FVC (fig. 2, table 4). As an example, the FEV1 data of one teenage male with $\mathrm{CF}$, homozygous for $\Delta \mathrm{F} 508$, was expressed according to four reference equations and is shown in figure 3 .

\section{Discussion}

A mathematical exercise was carried out by comparing how four ventilatory function reference equations affected the estimate of the development of FVC and FEV1 in young patients with CF. The conclusions are quite worrisome and confirm the hypothesis that the choice of reference equation would affect the estimate of lung-function development to an important degree. This study demonstrates, that a difference in the mathematical model of the equation directly affects the value and, more importantly, the estimated annual change of FVC and FEV1 in these patients with chronic lung disease.

The biological relationship between standing height and ventilatory function during puberty appears to be difficult, if not impossible, to describe accurately. Ideally, not only age but also pubertal stages should be included in the predictions, but this is not a practical option. To the best of the authors' knowledge, the only attempts to correct lung function appropriately for the pubertal growth spurt are the reported

Table 3. - Change of forced expiratory volume in one second (FEV 1 ) and forced vital capacity (FVC) during the study in a subgroup of 35 children aged $\geqslant 11$ yrs

\begin{tabular}{lrr}
\hline & \multicolumn{1}{c}{ FEV1 } & FVC \\
\hline$\%$ pred Z & $-1.8 \pm 3.5$ & $-0.4 \pm 3.0$ \\
$\%$ pred K & $3.5 \pm 6.3$ & $-0.6 \pm 3.2$ \\
$\%$ pred Q & $-2.4 \pm 3.2$ & $-1.2 \pm 2.7$ \\
$\%$ pred W & $-2.5 \pm 3.3$ & $-0.6 \pm 3.1$ \\
\hline
\end{tabular}

Data are presented as mean \pm SD unless otherwise stated. $\mathrm{Z}$ : using reference equation described in ZAPLETAL et al. [9]; $\mathrm{K}$ : using reference equation described in KNUDSON et al. [8]; Q: using reference equation described in QUANJER et al. [4]; $\mathrm{W}$ : using reference equation described in WANG et al. [6]. equations by QUANJER et al. [4] and by WANG et al. [6]. The equations by KNUDSON et al. [8] describing FVC or FEV1 as a linear function of height until the age of $12 \mathrm{yrs}$, and as a linear function of height and age from 12 yrs resulted in a significant mean increase of
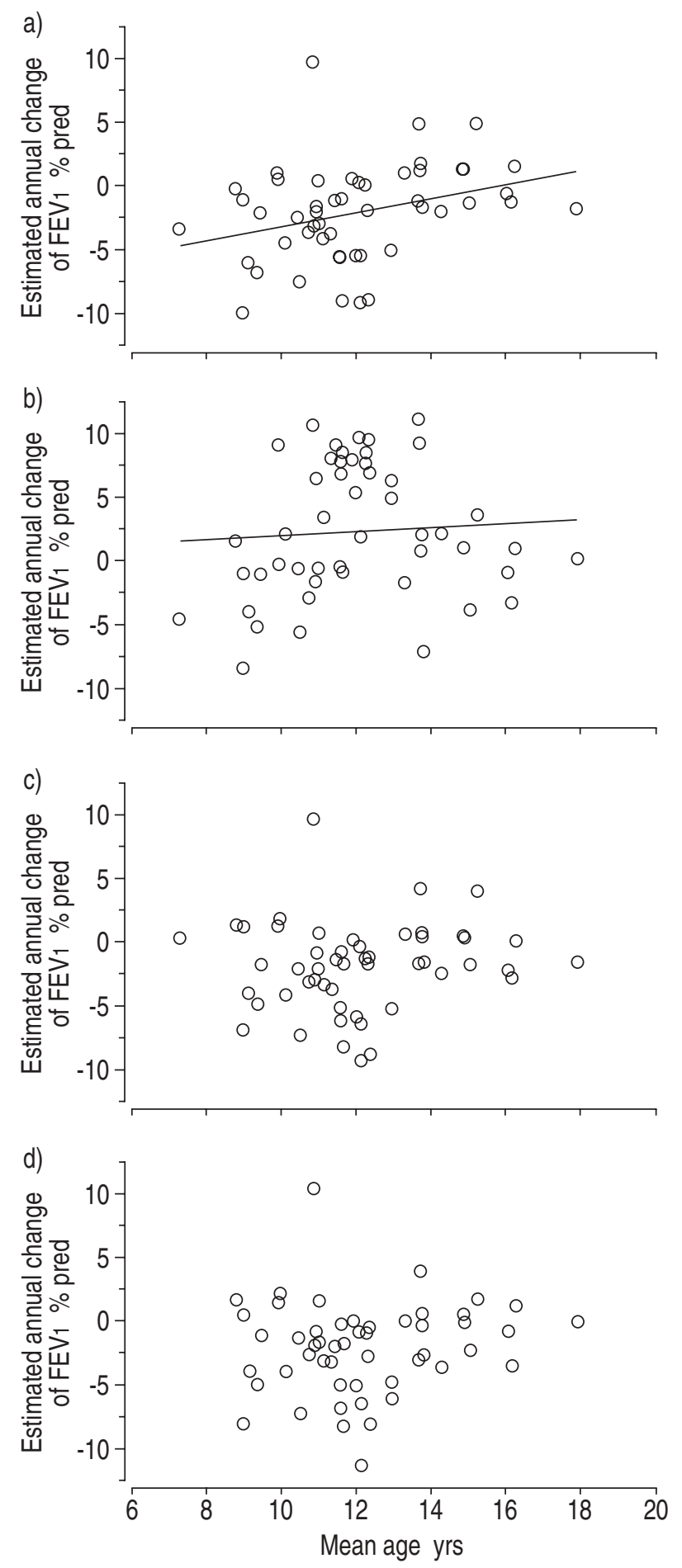

Fig. 1.-Relationship between mean individual age and individual annual change of forced expiratory volume in one second (FEV 1$)$ of 52 cystic fibrosis patients, expressed as $\%$ pred for four reference equations. a) ZAPLETAL et al. [9] $(\mathrm{r}=0.4, \mathrm{p}<0.01)$, b) KNUDSON et al. [8] $(\mathrm{r}=0.3, \mathrm{p}>0.05)$, c) QUANJER et al. [4] $(\mathrm{r}=0.09$, nonsignificant (NS)) and d) WANG et al. [6] ( $\mathrm{r}=0.09$, NS). 
Table 4. - Correlation coefficients ( $r$ ) and significant levels $(p)$ between mean individual ages and annual individual changes of forced expiratory volume in one second (FEV 1 ) or forced vital capacity (FVC) in 52 cystic fibrosis patients

\begin{tabular}{lccccc}
\hline & \multicolumn{2}{c}{ Change of FEV1 } & & \multicolumn{2}{c}{ Change of FVC } \\
\cline { 2 - 3 } \cline { 6 - 7 } & $\mathrm{r}$ & $\mathrm{p}$ & & $\mathrm{r}$ & $\mathrm{p}$ \\
\hline \% pred Z & 0.40 & 0.003 & & 0.31 & 0.03 \\
$\%$ pred K & 0.30 & 0.03 & & 0.05 & $\mathrm{NS}$ \\
$\%$ pred Q & 0.09 & $\mathrm{NS}$ & & 0.11 & $\mathrm{NS}$ \\
$\%$ pred W & 0.09 & $\mathrm{NS}$ & & 0.19 & $\mathrm{NS}$ \\
\hline
\end{tabular}

Z: using reference equation described in ZAPLETAL et al. [9]; $\mathrm{K}$ : using reference equation described in KNUDSON et al. [8]; Q: using reference equation described in QUANJER et al. [4]; $\mathrm{W}$ : using reference equation described in WANG et al. [6]; NS: nonsignificant.

FEV1 of $\sim 2 \%$ pred $\cdot \mathrm{yr}^{-1}$, and this was positively correlated with mean age. It seems highly unlikely that normalized airway calibre would improve with age in these CF patients because of the progressive lung-function impairment inherent to the chronic disease. It seems more likely that artifacts play a role, due to the limitations of most reference equations, as described earlier. The variable pattern of $\%$ pred $\mathrm{K}$ with age, with a peak near 12 yrs may be due to the subdivision of this reference population in one group of children younger and one older than 12 yrs. When FEV1 and FVC were expressed as a power function for height [9], annual changes of FEV1 and FVC were negative, as would be expected in CF, but were still positively correlated with age. Again, this is unlikely to be due to real improvement of lung disease and may still indicate that ventilatory function is underestimated during the pubertal growth spurt, and overestimated after or near the end of puberty. Thus, the hypothesized artifactual under and overestimation of lung function even plays a role in adolescents with $\mathrm{CF}$, despite the fact that their pubertal growth spurt is delayed and less pronounced [13, 14].

When reference equations, which were designed especially to limit "pubertal artifacts" [4, 6], were used mean annual changes of FEV1 and FVC (\% pred Q and $\%$ pred $\mathrm{W}$ ) were somewhat more negative compared to the other two equations, and this annual change was independent of age. This is not proof that these reference equations reflect reality better, but it suggests that the age-dependent artifacts are minimized. These findings are consistent with the study of ROSENFELD et al. [7]. The main difference between this study and that of RosENFELD et al. [7] is that this study also compared reference equations based on American and European children and adolescents. The present study demonstrates that the mean annual changes of FEV1 and FVC and their variance were similar for $\%$ pred $\mathrm{Q}$ and $\%$ pred $\mathrm{W}$, and that the reference equations by QUANJER et al. [4] and WANG et al. [6] only differed with respect to the absolute values. The values of FEV1 and FVC, expressed as \% pred W were systematically higher than when expressed as \% pred $\mathrm{Q}$. The authors speculate that this may have a biological background. These reference equations are
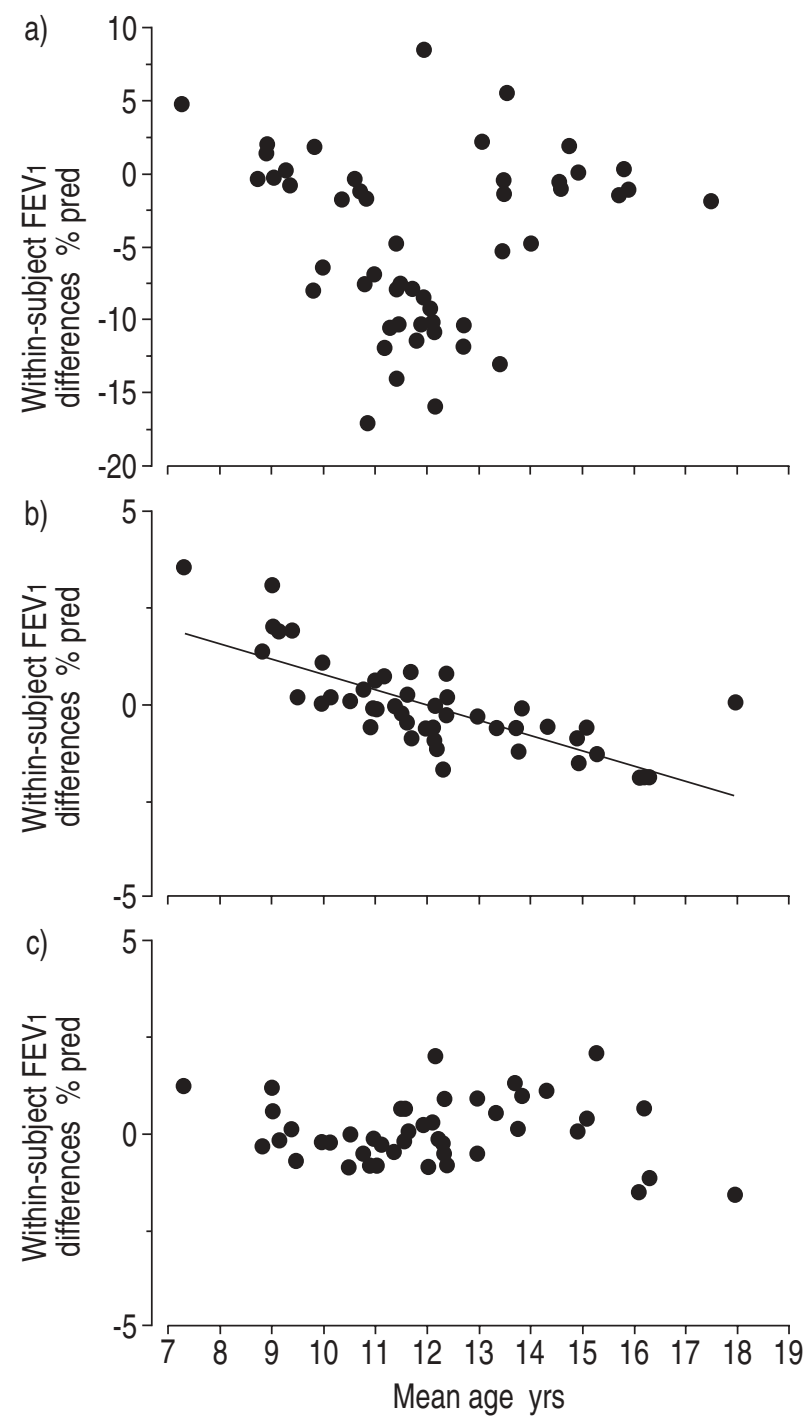

Fig. 2.-Within-subject differences of individual annual changes of forced expiratory volume in one second (FEV1) in 52 cystic fibrosis patients, according to reference equations used. a) Comparison of $\%$ pred $\mathrm{Q}$ and $\%$ pred $\mathrm{K}, \mathrm{b}) \%$ pred $\mathrm{Q}$ and $\%$ pred $\mathrm{Z}$ $(\mathrm{r}=0.98, \mathrm{p}<0.001), \mathrm{c}) \%$ pred $\mathrm{Q}$ and $\%$ pred $\mathrm{W}$. $\mathrm{Z}$ : using reference equation described in [9]; $\mathrm{K}$ : using reference equation described in [8]; Q: using reference equation described in [4]; W: using reference equation described in [6].

based on very large populations from the USA and from Europe that have no overlap. The conclusion may be that on average, the European population of children have larger lungs and airways for a given standing height, sex and age than the American children. Whether body proportions, especially leg length, are really different in these two populations remains to be investigated. Obviously, systematic differences are of less concern than age-dependent artifacts.

The comparisons were made with two "conventional" reference equations [8,9] because these were based on completely different mathematical models, and because both have been used in large studies to describe patients with asthma [10] and with CF [11, 12]. 

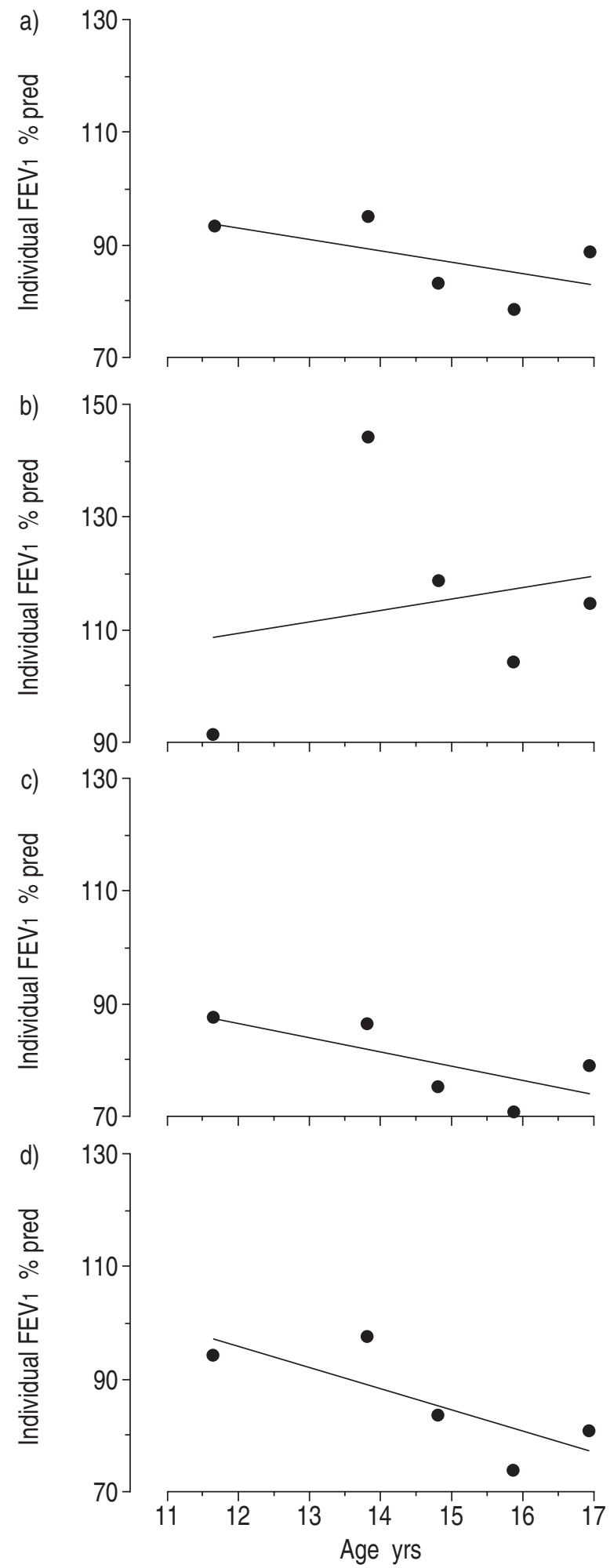

Fig. 3.-Illustration of individual trends for forced expiratory volume in one second (FEV1), according to four reference equations in a teenage male with cystic fibrosis. a) [9] $\left(-2.0 \% \mathrm{pred} \cdot \mathrm{yr}^{-1}\right)$, b) [8] $\left(2.0 \%\right.$ pred $\left.\cdot \mathrm{yr}^{-1}\right)$, c) [4] $\left(-2.6 \%\right.$ pred $\left.\cdot \mathrm{yr}^{-1}\right)$ and d) [6] $(-3.8 \%$ $\left.\mathrm{pred} \cdot \mathrm{yr}^{-1}\right)$.

It seems likely that other reference equations based on similar models, would have compared likewise.

This study focused on CF patients because they are a paediatric population in which reliable estimation of decline of function is an important clinical and research end-point, and the hypothesis was that even in this group the "pubertal artifacts" would play an important role. It is expected that these findings can be extrapolated to other respiratory disorders, because by studying differences between reference equations within subjects, the results of the evaluation are essentially independent of disease. Furthermore, in adolescents without severe chronic disease a more pronounced growth spurt can be expected, which implies that the pubertal artifacts described earlier will be even larger than in the patients with $\mathrm{CF}$.

The implications of these findings are important for patient care and for clinical research. For routine follow-up of patients, one should realize that FEV1 (mL) may be the most robust test of airway function, but apparently, longitudinal comparisons within growing individuals are quite difficult to interpret because correction for somatic and/or lung growth is inadequate. Hence, longitudinal reliability of FEV1 (\% pred) is not necessarily robust. Due to the "pubertal artifacts", most reference equations will tend to mask true deterioration, or inflate true improvement of FEV1 and FVC near the end of the growth spurt. As a result, discrepancies may develop between lung function (\% pred) and the clinical condition in children and adolescents with respiratory disease. These discrepancies should be taken into account when quality of care is assessed from estimated change of FEV1 (\% pred) as occurs for patients with $\mathrm{CF}$, and when results of different centres are compared. Figure 3 shows the longitudinal development of FEV1 (\% pred) of one patient according to four reference equations. This example illustrates that annual individual change of FEV1 (\% pred) may vary between 2 and $-3.8 \%$ pred. Hence, the choice of reference equation for FEV1 is likely to have a direct impact on the management of individual patients.

For scientific purposes these findings imply that interstudy comparisons are only reliable when no differences exist between mean age of the populations, and when the same reference equations are used. Even then, annual change of function can still be artificially age-dependent. Therefore, meta-analyses of longitudinal lung-function studies in children and adolescents should be performed preferably on raw data, using good quality reference equations. In randomized trials, differences in mean age between subgroups may affect outcome. This effect should be minimal after stratification for age. In long-term crossover trials the artifacts may lead to carry-over effects.

Guidelines for choosing appropriate reference equations have been published [3], but there have been no consensus reports recommending specific reference equations. The conclusions of the present study imply that age and height are needed as independent variables to improve the quality of paediatric pulmonary reference equations, and that updating these equations is probably necessary because of cohort effects in the age-height relationship in children and adolescents. The suggestion is that large international bodies such as the American Thoracic Society and the European Respiratory Society 
should recommend, and regularly update, specific reference equations to be used for older children and adolescents or develop them, based on large databases. The reference equations for forced expiratory volume in one second and forced vital capacity developed by QUANJER et al. [4] and those by WANG et al.[5] may be a suitable starting point.

\section{References}

1. Glindmeyer HW, Diem JE, Jones RN, Weill H. Noncomparability of longitudinally and cross-sectionally determined annual change in spirometry. Am Rev Respir Dis 1982; 125: 544-548.

2. Pattishall EN, Helms RW, Strope GL. Noncomparability of cross-sectional and longitudinal estimates of lung growth in children. Pediatr Pulmonol 1989; 7: 22-28.

3. Quanjer PH, Stocks J, Polgar G, Wise M, Karlberg J, Borsboom G. Compilation of reference values for lung function measurements in children. Eur Respir J 1989; 2: $184 \mathrm{~S}-262 \mathrm{~S}$.

4. Quanjer PH, Borsboom GJJM, Brunekreef B, et al. Spirometric reference values for white European children and adolescents: Polgar Revisited. Pediatr Pulmonol 1995; 19: 135-142.

5. Schrader PC, Quanjer PH, van Zomeren BC, Wise ME. Changes in the FEV1-height relationship during pubertal growth. Bull Eur Physiopathol Respir 1984; 20: $381-388$.

6. Wang X, Dockery DW, Wypij D, Fay ME, Ferris BG. Pulmonary function between 6 and 18 years of age. Pediatr Pulmonol 1993; 15: 75-88.

7. Rosenfeld M, Pepe MS, Longton G, Emerson J,
FitzSimmons S, Morgan W. Effect of choice of reference equation on analysis of pulmonary function in cystic fibrosis patients. Pediatr Pulmonol 2001; 31: 227-237.

8. Knudson RJ, Lebowitz MD, Holberg CJ, Burrows B. Changes in the normal maximal expiratory flowvolume curve with growth and aging. Am Rev Respir Dis 1983; 127: 725-734.

9. Zapletal A, Paul T, Samanek M. Die bedeutung heutiger methoden der lungenfunktionsdiagnostik zur feststellung einer obstruktion der atemwege bei kindern und jugendlichen. Z Erkrank Atm Org 1977; 149: 343-371.

10. van Essen-Zandvliet EEM, Hughes MD, Waalkens $\mathrm{HJ}$, et al. Effects of 22 months treatment with inhaled corticosteroids and/or beta2-agonists on lung function, airway responsiveness and symptoms in children with asthma. Am Rev Respir Dis 1992; 146: 547-554.

11. Ramsey BW, Pepe MS, Quan JM, et al. Intermittent administration of inhaled tobramycin in patients with cystic fibrosis. $N$ Engl J Med 1999; 340: 23-30.

12. Fuchs HJ, Borowitz DS, Christiansen DH, et al. Effect of aerosolized recombinant human DNase on exacerbations or respiratory symptoms and on pulmonary function in patients with cystic fibrosis. $N \mathrm{Engl}$ $J$ Med 1994; 331: 637-642.

13. Byard PJ. The adolescent growth spurt in children with cystic fibrosis. Ann Hum Biol 1994; 21: 229-240.

14. Haeusler G, Frisch H, Waldhor T, Gotz M. Perspectives of longitudinal growth in cystic fibrosis from birth to adult age. Eur J Pediatr 1994; 153: 158-163.

15. Quanjer PH, Tammeling GJ, Cotes JE, Pedersen OF, Peslin R, Yernault J. Lung volumes and forced ventilatory flows. Eur Respir J Suppl 1993; 16: 5-40. 